doi: $10.2306 /$ scienceasia1513-1874.2014.40.405

\title{
Cloning, characterization, and heterologous expression of a dextranase gene from Penicillium pinophilum SMCU3-14
}

\author{
Panan Rerngsamran*, Pimonrut Temjitpukdee, Nilnate Assavasirijinda, Supat Chareonpornwattana, \\ Suthep Thaniyavarn
}

Department of Microbiology, Faculty of Science, Chulalongkorn University, Payathai, Bangkok 10330 Thailand

*Corresponding author, e-mail: panan.r@chula.ac.th

Received 31 Mar 2014

Accepted 11 Nov 2014

\begin{abstract}
A DNA fragment encoding dextranase was cloned from Penicillium pinophilum SMCU3-14 using the genome walking approach. Sequence analysis of the gene (SMCU-DEX) revealed a putative CAAT box at - 165 (CCAAT), a putative TATA box at -93 (TATAA) in the $5^{\prime}$-noncoding region, and a polyadenylation signal (AATAAG) in the $3^{\prime}$-noncoding region. A cDNA sequence analysis revealed no evidence of introns. The deduced open reading frame is 1824 bp in length and encodes a predicted protein of 608 amino acids (molecular weight (MW) of $\sim 66 \mathrm{kDa}$ ), with a putative $\mathrm{N}$-terminal 20 -amino acid signal peptide, giving a predicted mature protein of 588 amino acids (MW of $\sim 64 \mathrm{kDa}$ ) that belongs to glycosyl hydrolase family 49 , as with other fungal dextranases. This is the first report of a dextranase gene sequence from P. pinophilum. The cDNA was cloned and expressed in Escherichia coli, and the transformants showed dextranase activity on a dextran-containing agar medium. Crude extracts from the transformants analysed by sodium dodecyl sulphate polyacrylamide gel electrophoresis containing blue dextran revealed a distinct specific band of dextranase activity at an MW of approximately $66 \mathrm{kDa}$. This recombinant dextranase is likely to have valuable and cost-effective applications in medicine and industry.
\end{abstract}

KEYWORDS: genome walking, Escherichia coli

\section{INTRODUCTION}

Dextran is a homopolysaccharide composed of Dglucose units connected by $\alpha-1,6$ glucosidic linkages and various numbers of side branches joined to the backbone via $\alpha-1,2, \alpha-1,3$, or $\alpha-1,4$ linkages. Dextran is typically synthesized from sucrose by the action of dextransucrase or glucosyltransferase enzymes. In the sugar industry, sugarcane juice, the raw material for sugar production is always contaminated with bacteria of the genera Lactobacillus, Leuconostoc, or Streptococcus, which produce an extracellular dextransucrase that synthesizes dextran from the sucrose present in the juice ${ }^{1,2}$. This formation of dextran therefore causes decreases in the yield and quality of the sugar produced; clogs and blocks the pipelines, tanks, strainers, and filters; increases the viscosity of the juice; results in a poor clarification and filtration ability; and further inhibits sucrose production (extraction) by decreasing the rate of sucrose crystallization $^{3}$. For these reasons, dextran production is a considerable problem resulting in severe economic loss to the sugar industry.

In addition, dextran is also a problem for dental health. Oral streptococci, which colonize the tooth surface, synthesize dextran from sucrose using glucosyltransferases ${ }^{4}$. Dextran is a structural component of dental plaque, a component of closely packed bacteria and noncellular materials, which causes the development of dental caries 5 .

Physicochemical techniques to remove dextran have proven to be unsuccessful. Enzymes that specifically hydrolyse dextran are therefore employed to overcome some of the problems associated with the removal of dextran ${ }^{6}$.

Dextranase (EC 3.2.1.11, $\alpha$-1,6 glucan 6-glucanohydrolase) is an inducible enzyme that specifically hydrolyses the $\alpha$-1,6-glucosidic linkages within the dextran molecule to release smaller oligosaccharides ${ }^{7}$. As this process reversibly reduces the stickiness caused by dextran, it has been suggested as a mean to solve the problem of microbe-produced dextran in the cane sugar industry ${ }^{8}$. The same rationale has also been used for the prevention of caries by inhibiting 
biofilm formation on teeth ${ }^{9,10}$, whereby dextranase retards plaque deposition on the tooth surface, thus repressing the adherence of oral streptococci to the tooth surface and inhibiting the formation of dental caries. Moreover, dextranase has been used in degrading dextran to an appropriate smaller size range suitable for use as a synthetic blood volume expander in patients suffering from hypovolaemic shock ${ }^{11}$. The use of dextranase has also been proposed as a method to increase antibiotic efficacy in infective endocarditis and a universal targeting method for therapeutic agents ${ }^{12}$. Sources of dextranases are widespread: the enzyme has been found in mammalian tissues and in various microorganisms, such as fungi, yeasts, bacteria, and Actinomyces ${ }^{13,14}$. Fungi are the most important commercial source of dextranases as they produce high levels of enzyme ${ }^{15}$. Although several fungi have been reported to produce dextranase, only a few nucleotide sequences of fungal dextranases are available in public databases.

Conserved regions in the amino acid sequences of dextranases from different sources have been reported ${ }^{16}$. On the basis of their amino acid sequence similarity, dextranases have been classified into two glycosyl hydrolase families (GH), GH49 and GH66, among which there is no significant sequence similarity. The bacterial dextranases from Streptococcus species belong to the GH66 family ${ }^{17}$, whereas the bacterial dextranases from Arthrobacter species, fungal dextranases from Penicillium species, and the dextranase from the yeast Lipomyces starkeyi all belong to the GH49 family ${ }^{18,19}$.

In addition to their high levels of enzyme, fungi are also the most important source for commercial dextranase production due to the stability of the enzymes at high temperatures. However, because fungi also produce various antibiotics and metabolites, there has been difficulty in obtaining consumer approval ${ }^{20}$. To solve this problem, a fungal dextranase gene can be heterologously expressed in an alternate host, such as a non-harmful bacterial species. Previous studies have shown that Penicillium pinophilum SMCU3-14 produces a high level of dextranase activity, up to 600 units $/ \mathrm{ml}^{21}$. In the present report, cloning and sequence characterization of a dextranase-encoding genomic DNA and cDNA from this fungus and its expression in Escherichia coli were described.

\section{MATERIALS AND METHODS}

\section{Strains and vectors}

P. pinophilum SMCU3-14 (EU635728), previously isolated from a local soil sample ${ }^{21,22}$, was used as
Table 1 Primers used in this study.

\begin{tabular}{ll}
\hline Primers & Sequences $\left(5^{\prime}\right.$ to $3^{\prime}$ direction) \\
\hline Dex1F & TCTGTACCTGGTGGCATGATTC \\
Dex1R & TCGCTAATTTGACTTGAGATGC \\
Dex5R & ACGCAGTACCATGTTTGACCACCCCAAGG \\
Dex6R & TGCAACATAGTTGTCGCCGGCATTGGCTTG \\
Dex3F & GCTGCGCCAATCTCACAAGTATTCCGTCCGTGC \\
Dex4F & CTTTGTATATGAATCGATCCCCCGGAACG \\
Dex8F & TAATTGACGCCTTGACAGGTGATG \\
Dex10R & CATATCGTCCACGTCTTTTGAAAG \\
Dex14F & CTCAAACATATGCCCACAATG \\
Dex14R & AGCTGGAGGATCCCTAATCTG \\
\hline
\end{tabular}

$\dagger$ Underlined sequences of Dex14F and Dex14R indicate the $N d e \mathrm{I}$ and Bam $\mathrm{HI}$ restriction sites, respectively.

a source for the isolation of the dextranase gene. Vectors pDrive (Qiagen, USA) and pJET1/blunt (Fermentas, USA) were used to clone the PCR products. pETHIS, kindly provided by National BioResource Project (NIG, Japan), was used as an expression vector. E. coli isolate DH5 $\alpha$ was used for all the cloning experiments whereas E. coli Rosetta-gami B (DE3)pLysS (Novagen, USA) was used to express recombinant dextranase protein. All the standard molecular genetic techniques were performed according to Sambrook and Russell ${ }^{23}$.

\section{Cloning of the dextranase gene from $P$. pinophilum SMCU3-14}

Chromosomal DNA of P. pinophilum SMCU3-14 was isolated according to Reader and Broda ${ }^{24}$. A segment of the dextranase gene was amplified based on the conserved sequences of different dextranase genes among Penicillium. To this end, the Dex1F/Dex1R primers (Table 1) were designed and used to amplify the central part of the dextranase gene using $P$. pinophilum SMCU3-14 genomic DNA as a template. The PCR product was commercially sequenced bidirectionally by 1 st Base Laboratory, Malaysia.

Gene-specific primers were designed based on the obtained sequence to amplify the $5^{\prime}$ and $3^{\prime}$ sequences flanking the conserved sequence using the BD Genome Walker Universal kit (BD Biosciences Clontech, USA). To amplify the $5^{\prime}$ flanking sequence, outer and nested reverse primers, Dex5R and Dex6R (Table 1), specific to the dextranase conserved sequences were designed; and outer and nested forward primers Dex3F and Dex4F (Table 1) were used to amplify the $3^{\prime}$ flanking sequence. These primers were used together with the outer adaptor primer 1 (AP1) and nested adaptor primer 2 (AP2) provided in the kit in accordance with the manufacturer's protocol. Briefly, P. pinophilum SMCU3-14 genomic DNA was separately digested with the DraI, EcoRV, PvuII, and $S t u I$ blunt-ending restriction enzymes and then sep- 
arately ligated to the BD Genome Walker Adaptor to form four restriction libraries. The upstream and downstream flanking fragments of the conserved dextranase sequence (via PCR amplification using the Dex1F/Dex1R primer pair) were then amplified by primary PCR using the Dex5R/AP1 and Dex3F/AP1 primer pairs, respectively. The primary PCR reaction cycles using a GeneAmp PCR System 2400 (Perkin Elmer, USA) thermal cycler were as follows: 7 cycles at $94{ }^{\circ} \mathrm{C}$ for $2 \mathrm{~s}$ and $70^{\circ} \mathrm{C}$ for $3 \mathrm{~min}$, followed by 32 cycles at $94{ }^{\circ} \mathrm{C}$ for $2 \mathrm{~s}$ and $69^{\circ} \mathrm{C}$ for $3 \mathrm{~min}$, and a final extension at $67^{\circ} \mathrm{C}$ for $4 \mathrm{~min}$.

The primary PCR products were then diluted 50-fold and used as the template for the secondary nested PCR. This secondary amplification step served to specifically amplify the desired product prior to cloning and utilized the nested reverse (Dex6R) and adaptor (AP2) primers and the nested forward (Dex4F) and adaptor (AP2) primers to amplify the $5^{\prime}$ and $3^{\prime}$ flanking fragments, respectively. The secondary PCR reaction cycles using a DNA Thermal Cycle 2400 (Perkin Elmer) instrument were as follows: 5 cycles at $94^{\circ} \mathrm{C}$ for $2 \mathrm{~s}$ and $70^{\circ} \mathrm{C}$ for $3 \mathrm{~min}$, followed by 20 cycles at $94^{\circ} \mathrm{C}$ for $2 \mathrm{~s}$ and $60^{\circ} \mathrm{C}$ for $3 \mathrm{~min}$, and a final extension at $67^{\circ} \mathrm{C}$ for $4 \mathrm{~min}$.

After the secondary amplification, the PCR products were purified and cloned into the pDrive Cloning Vector (Qiagen, USA). The presence of the insert was confirmed by restriction enzyme digestion, and the recombinant plasmids were extracted and commercially sequenced in both directions, as above. The sequenced fragments were assembled and characterized using DNASIS (Hitachi Software Engineering, USA). Sequence alignments were used to search GenBank public database sequences with the online BLASTN algorithm (www.ncbi.nlm.nih.gov).

To obtain the complete dextranase gene in a single fragment by PCR amplification, the Dex8F/Dex10R primers (Table 1) were used with $P f u$ DNA polymerase (Fermentas, USA) and P. pinophilum SMCU314 genomic DNA as the template. After amplification, the PCR product was purified and cloned into the pJET/blunt-cloning vector (Fermentas, USA). The recombinant plasmid harbouring the PCR product encoding the complete dextranase gene was then sequenced and analysed as described before.

\section{Cloning of the dextranase cDNA of $P$. pinophilum SMCU3-14 (SMCU-DEX)}

P. pinophilum SMCU3-14 was cultivated in $50 \mathrm{ml}$ of modified Fukumoto's medium ${ }^{25}$, with or without $1 \%(\mathrm{w} / \mathrm{v})$ dextran (industrial grade; Sigma, USA) to induce dextranase production, at room temperature with shaking at $200 \mathrm{rpm}$. Mycelium samples were harvested by filtration every three days, lyophilized until completely dry, and kept at $-70^{\circ} \mathrm{C}$ until use. Total RNA was extracted using the e-Zi RNA Extraction kit (Sunolin, Thailand) according to the instructions provided.

Total RNA from each sample was separated by formaldehyde agarose gel electrophoresis and transferred to a Hybond $\mathrm{N}$ membrane according to the manufacturer's protocol (Amersham Biosciences, USA). The conserved dextranase fragment obtained by PCR amplification with the Dex1F/Dex1R primers was used as a probe in a Northern hybridization assay using the DIG High Prime DNA Labelling and Detection Starter Kit I (Roche Biochemical Products, Germany), as described by the manufacturer.

Poly $(\mathrm{A})^{+}$RNA was isolated from dextran-induced mycelia by the PolyATtract mRNA Isolation System III (Promega, USA), and cDNA was synthesized with $P f u$ DNA polymerase using the Universal RiboClone cDNA Synthesis System (Promega, USA) according to the manufacturer's specifications. The DNA fragment encoding the full-length dextranase gene from the start to stop codons was obtained by PCR using the cDNA and Dex14F/Dex14R primer pair (Table 1), containing a $5^{\prime}$ flanking $N d e I$ or BamHI restriction sites (Table 1, underlining indicates the NdeI and BamHI restriction sites, respectively). The PCR product was blunt-end cloned into the pJET1/blunt Cloning Vector (Fermentas, USA), and the recombinant plasmid (pNA-1) was commercially sequenced, as detailed above.

Sequence analyses were performed using DNASIS, BLASTN, BLASTX, and ClustalX. For the purpose of comparison, the sequences of dextranases and related proteins from Arthrobacter sp. (accession number P39652), Arthrobacter globiformis (accession number BAA13598), Brevibacterium fuscum var. dextranlyticum isomaltotriodextranase (accession number BAA76382), Penicillium minioluteum (accession number AAB47720), and Aspergillus niger (accession number BAA18971) were used.

\section{Southern hybridization of the dextranase gene}

Aliquots $(10 \mu \mathrm{g})$ of $P$. pinophilum SMCU3-14 genomic DNA were digested with BamHI, EcoRI, HindIII (NEB Biolabs, USA), PstI (Fermentas, USA), $K p n \mathrm{I}$, or $\mathrm{Xba \textrm {I }}$ (Promega, USA) restriction enzymes. Each digestion was resolved on a $1 \%(\mathrm{w} / \mathrm{v})$ agaroseTBE gel and then transferred to a Hybond $\mathrm{N}$ membrane (Amersham Biosciences, USA). The complete dextranase gene ( $\sim 3 \mathrm{~kb}$ PCR product) was used as the probe for Southern hybridization using the DIG 
High Prime DNA Labelling and Detection Starter Kit I (Roche Biochemical Products, Germany), as described by the manufacturer. Genomic DNA from Lentinus sp. was used as a negative control.

\section{Expression of recombinant dextranase protein}

To construct the plasmid for the heterologous expression of recombinant dextranase in E. coli, pNA-1 was digested with $N d e \mathrm{I}$ and BamHI. The released dextranase gene fragment was gel purified and directionally cloned into the corresponding NdeI and BamHI sites of the pETHIS expression vector. The obtained recombinant plasmid (pNAT2) was transformed into E. coli Rosetta-gami B (DE3)pLysS, and the transformants screened on LB agar supplemented with $100 \mu \mathrm{g} / \mathrm{ml}$ ampicillin and $34 \mu \mathrm{g} / \mathrm{ml}$ chloramphenicol. Protein expression was induced with $4 \mathrm{mM}$ Isopropyl $\beta$-D-1-thiogalactopyranoside (IPTG).

Fifteen colonies of transformants and the host strain carrying the empty pETHIS vector as a control were spotted onto Luria-Bertani (LB) agar plates containing $1 \%(\mathrm{w} / \mathrm{v})$ dextran and $10 \mathrm{mM}$ IPTG to test for the production of extracellular dextranase activity. After $18 \mathrm{~h}$ of incubation at $37^{\circ} \mathrm{C}$, the test plate was overlaid with $95 \%(\mathrm{v} / \mathrm{v})$ ethanol to reveal the cloudy dextran and observe any dextranase activity as a clear halo zone around the positive colonies.

Dextranase activity was also zymographically assayed by sodium dodecyl sulphate (SDS) polyacrylamide gel electrophoresis (SDS-PAGE) containing blue dextran (D5751; Sigma, USA). Briefly, E. coli Rosetta-gami B (DE3)pLysS cells transformed with pNAT2 and the host strain carrying the empty vector were cultured with and without IPTG induction. The crude protein extracts were resolved by SDS-PAGE $(10 \%(\mathrm{w} / \mathrm{v}) \text { acrylamide resolving gel })^{26}$ containing $0.5 \%(w / v)$ blue dextran. The resolved gel was incubated overnight at $55^{\circ} \mathrm{C}$ in $0.05 \mathrm{M}$ sodium acetate buffer ( $\mathrm{pH} 4.5$ ) containing $1 \%$ (v/v) Triton X-100 to remove the SDS and allow the renaturation of the enzyme and the dextranase cleavage of blue dextran ${ }^{27}$. Dextranase activity was observed as a clear band against a blue background. Duplicate samples were separated by SDS-PAGE without blue dextran and then stained with Coomassie brilliant blue to visualize the protein and allow an estimate of the (subunit) molecular weight.

\section{RESULTS}

\section{Cloning of the genomic dextranase gene}

A 1-kb fragment of the dextranase gene was isolated using PCR amplification (Dex1F/R primers)

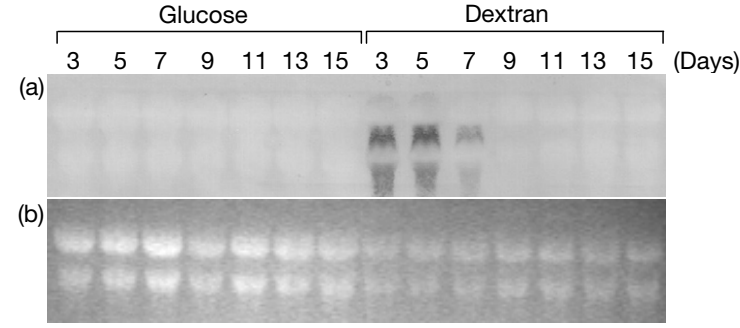

Fig. 1 (a) Northern hybridization of the total RNA isolated from $P$. pinophilum SMCU3-14 grown in a medium containing 3\% (w/v) glucose with or without $1 \%(\mathrm{w} / \mathrm{v})$ dextran at the time points indicated using SMCU-DEX as a probe. (b) Total RNA visualized as an ethidium-bromide stained gel prior to transfer and Northern blotting.

from P. pinophilum SMCU3-14 and commercially sequenced. The consensus DNA sequence was then used to BLASTN search against the GenBank database and showed $100 \%$ DNA sequence identity to five reported dextranase genes from Penicillium spp. Thus it was concluded that a portion of the dextranase gene of $P$. pinophilum SMCU3-14 (SMCU-DEX) had been isolated.

The complete genomic DNA encoding the dextranase and the $5^{\prime}$ and $3^{\prime}$ flanking sequences were then obtained by genome walking using the BD GenomeWalker universal kit. A fragment of approximately $3.0 \mathrm{~kb}$ was cloned and sequenced. The sequence was verified as a dextranase gene by homology searching (BLASTN) of the GenBank database, with the highest DNA sequence identity (99\%) being found to the dextranase gene of closely related $P$. minioluteum (accession number L41562). The complete nucleotide sequence of $S M C U-D E X$ was deposited in the GenBank database under the accession number EU635729.

\section{Northern hybridization and cDNA cloning of the dextranase gene}

Total RNA extracts from $P$. pinophilum SMCU314 grown in Fukumoto's media with or without 1\% (w/v) dextran (to induce dextranase production) were harvested every two days, and the samples were subjected to a Northern analysis using a complete $S M C U$ $D E X$ fragment as the probe. The result revealed that dextranase was only expressed in the dextraninduced culture; the expression was transient, starting as early as after 3 days of incubation, reaching the highest level at day 5, and then decreasing until it was undetectable by day 9 and thereafter (Fig. 1). Accordingly, the total RNA sample from the 5-day post- 
dextran-induced culture was selected for subsequent cDNA amplification by PCR using the gene-specific DEX14F/R primers. The obtained cDNA was cloned and commercially sequenced.

\section{Sequence characterization of the complete dextranase gene of $P$. pinophilum SMCU3-14}

The genomic and cDNA sequences (Fig. 2) were analysed using the DNASIS program. Both sequences were verified as a dextranase gene by homologous analysis with other fungal dextranase genes available in GenBank. No evidence of any intron in $S M C U$ $D E X$ was found.

Four potential initiation codons $\left(\mathrm{ATG}_{1}, \mathrm{ATG}_{2}\right.$, $\mathrm{ATG}_{3}$, and $\mathrm{ATG}_{4}$ in Fig. 2) were found, all of which coded for the same protein that differed only in the length of the $\mathrm{N}$-terminus $(12,31$, and 34 amino acids smaller for $\mathrm{ATG}_{2}, \mathrm{ATG}_{3}$, and $\mathrm{ATG}_{4}$, respectively, than for $\left.\mathrm{ATG}_{1}\right)$. Translation from the first ATG codon $\left(\mathrm{ATG}_{1}\right)$, for a dextranase gene (SMCU-DEX) sequence from position 1450-3273, would generate a deduced 1824-bp open reading frame (ORF) that encodes a protein of 608 amino acids (SMCUDEX) with a predicted molecular weight $\left(\mathrm{M}_{\mathrm{W}}\right)$ of approximately $66 \mathrm{kDa}$. This polypeptide contained a predicted (by SIGNALP version 3.0; www.cbs.dtu.dk/ services/SignalP) $\mathrm{N}$-terminal sequence of 20-amino acid residue signal peptide (positions $1-20$ in the ORF) (Fig. 2), resulting in a predicted mature protein of 558 amino acids and an $\mathrm{M}_{\mathrm{W}}$ of $\sim 64 \mathrm{kDa}$.

Compared to other dextranases obtained from GenBank by BLASTX and BLASTP searches, SMCU-DEX revealed $97 \%$ amino acid sequence identity to the dextranase from $P$. funiculosum (accession number AJ272066) and 99.5\%, 90\%, and 87\% identity to the enzymes from three isolates of $P$. minioluteum (accession numbers L41562, AF020619, and DQ394070; see Garcia et al $^{28}$, Li et al ${ }^{19}$ ).

The deduced amino acid sequence was compared to the GenBank conserved domain and amino acid databases, which revealed that SMCU-DEX belongs to the GH49 family. Furthermore, SMCUDEX shared the same seven conserved amino acid regions as other GH49 dextran-hydrolysing enzymes, although the sequence in region III exhibited a higher degree of identity (12/16 residues, $75 \%)$ than the other six regions (39-58\% identity) (Fig. 3).

Neural network promoter prediction version 2.2 (www.fruitfly.org/seq_tools/promoter.html) was used to identify any putative promoter sequence upstream of $\mathrm{ATG}_{1}$. A CAAT Box-like sequence (CCAAT) at position 1281-1285, a TATAA box at position 1353-1357, and a putative transcription start site at position 1385 were found (Fig. 2). In addition, the Hamming clustering polyA prediction in eukaryotic genes (HC polyA) program (http://bioinfo4.itb.cnr.it/ $\sim$ webgene/wwwHC_polya.html) revealed a putative polyA signal-like sequence (AATAAG) at position 3490-3495 (Fig. 2).

\section{Determination of the $S M C U$-DEX gene copy number in P. pinophilum SMCU3-14}

To determine the likely copy number of the $S M C U$ $D E X$ gene in the genome of $P$. pinophilum SMCU314 , Southern hybridization was performed using the full-length $S M C U-D E X$ sequence as the hybridization probe (Fig. 4). All the restriction-digested DNA preparations (BamHI, EcoRI, HindIII, PstI, KpnI, and $X b a \mathrm{I})$ produced a hybridization band, whereas no signal was observed with Lentinus sp. genomic DNA (negative control). Furthermore, all samples showed only a single hybridization band, except for the $\mathrm{Xba \textrm {I }}$ restricted DNA, which yielded two smaller fragments. The most likely explanation was that there is a single copy of SMCU-DEX in the P. pinophilum SMCU314 genome but that it contains an internal $X b a \mathrm{I}$ site; an analysis of the $S M C U-D E X$ sequence revealed a $X b a \mathrm{I}$ site in the $5^{\prime}$ flanking region (see 1218-1224 bp in Fig. 2).

\section{Heterologous expression of recombinant dextranase in $E$. coli}

The SMCU-DEX gene was subcloned from pNA-1 into pETHIS at the NdeI and BamHI sites to yield the recombinant plasmid pNAT2. The plasmid was propagated in E. coli $\mathrm{DH} 5 \alpha$ and then transformed into E. coli Rosetta-gami B (DE3)pLysS for dextranase expression under the control of the strong T7 promoter with IPTG induction. Transformants were plated onto LB plates with $1 \%(w / v)$ dextran; after $18 \mathrm{~h}$ of growth, the plate was overlaid with $95 \%$ (v/v) ethanol to visualize the dextran and screen for dextranase activity. All the screened transformants bearing pNAT2 revealed a clear halo zone around the colony demonstrating that they produced dextranase (Fig. 5), whereas the E. coli transformed with the empty pETHIS vector did not produce any halo zone.

\section{In-gel dextranase activity assays}

To confirm the expression and activity of dextranase in the transformant, an in-gel zymograph for dextranase activity was performed. Supernatants from IPTGinduced and non-induced cultures of one selected transformant (pNAT2-transformed E. coli Rosettagami B (DE3)pLysS) and the host strain transformed 


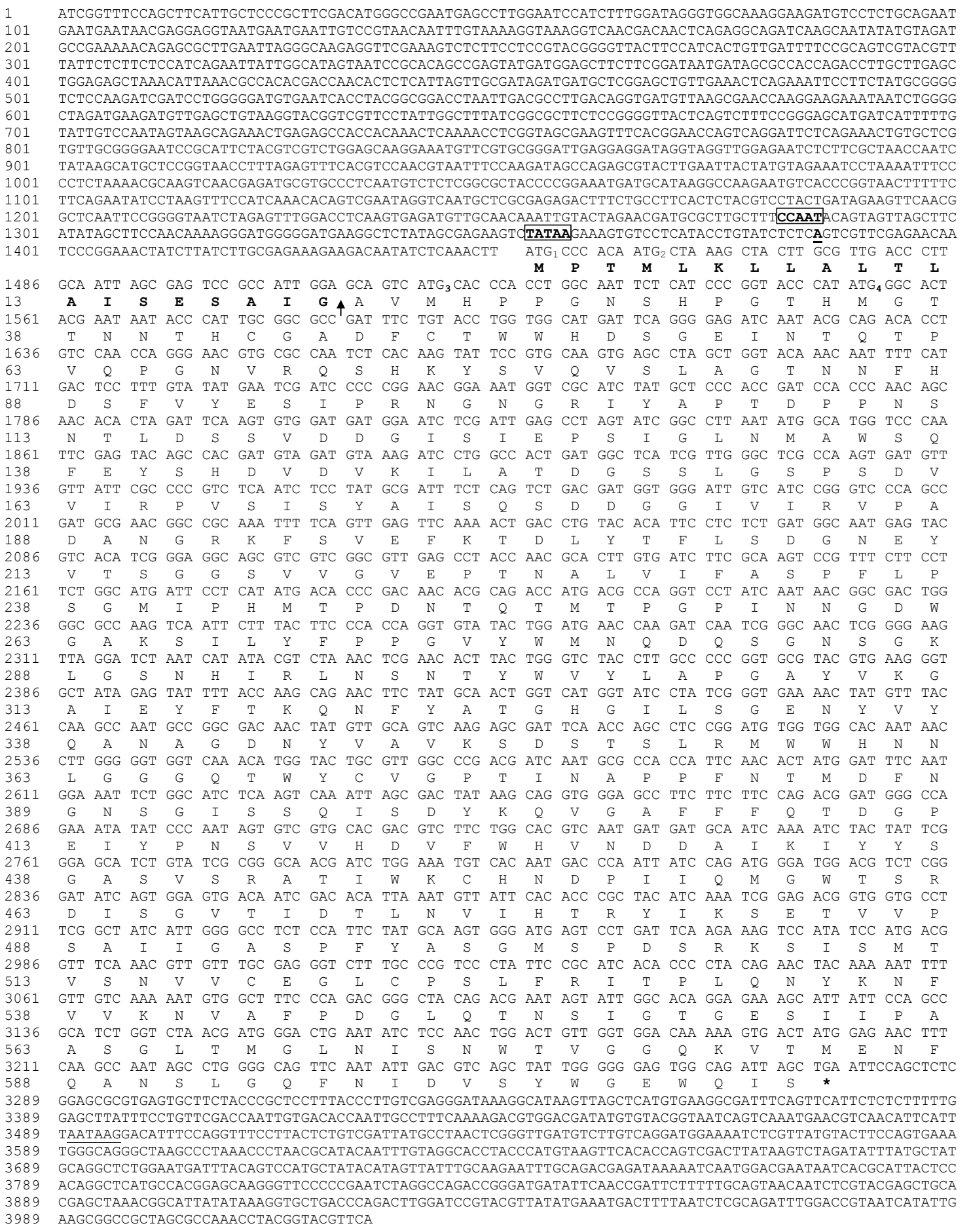

Fig. 2 The nucleotide sequence of the $S M C U-D E X$ gene and its deduced amino acid sequence. Four putative initiation codons $\left(\mathrm{ATG}_{1-4}\right)$ are shown. The putative CAAT-box and TATA-box of the promoter are boxed. The $\underline{\mathbf{A}}$, in bold and underlined, indicates the potential transcription start site. The amino acid sequence at positions 1-20 (from $\mathrm{ATG}_{1}$ ) in bold are the putative $\mathrm{N}$-terminal signal peptide residues, with the signal peptidase cleavage site marked with an arrow $(\uparrow)$. After the stop codon $(\mathrm{TGA} ; *)$, the underlined AATAAG nucleotide sequence indicates the putative polyA signal sequence. This sequence has been submitted to GenBank database under accession number EU635729. 


\begin{tabular}{|c|c|c|c|c|c|c|c|}
\hline Source & Region I & Region II & Region III & Region IV & Region V & Region VI & Region VII \\
\hline Arthro-1 & TWWHDNGV & GYRFSVEFEPQLYT & WVYLAPGAYVKGAFRF & TGYGVLSGEQYVY & YKQVGSWYWQTDGIELYKG & NDDVLKMYHSDVT & PVIQWGWTPRNI \\
\hline Arthro-2 & TWWHDNGV & GYRESVEFEPQLYT & WVYLAPGAYVKGAFRF & TGYGVLSGEQYVY & YKQVGSWYWQTDGIELYQG & NDDVLKMYHSDVS & PVVQWGWTPRNI \\
\hline IMTD & TWWHDNAV & GYRFSVEFDPQLYT & WVYLAPGAYVKGAFRF & TGYGVLSGEQYVY & YKQVGSWYWQTDGIELYQG & NDDVLKMYHSDVT & PVIQWGWTPRNI \\
\hline SMCU-DEX & TWWHDSGE & GRKFSVEFKTDLYT & WVYLAPGAYVKGAIEY & TGHGI LSGENYVY & YKQVGAFFFQTDGPEIYPN & NDDAIKIYYSGAS & PI IQMGWTSRDI \\
\hline PUDEX & TWWHDSGE & GRKFSVEFKTDLYT & WVYLAPGAYVKGAIEY & TGHGILSGENYVY & YKQVGAFFFQTDGPEIYPN & NDDAIKIYYSGAS & PI IQMGWTSRDI \\
\hline IPUA & TWWHNTGE & GYRFSVEFDDDLIS & WVYFAPGAYVKGAVEF & SGHGVLSGEQYVW & YKQVGAFYGQTDGLEMYPG & DDDGLKMYYSNVT & PVVEFGWTPRNT \\
\hline
\end{tabular}

Fig. 3 The seven conserved regions (I-VII) of dextranases, as reported by Aoki and Sakano ${ }^{16}$, are indicated. Arthro-1, Arthrobacter sp. CB-8 endodextranase (P39652); Arthro-2, A. globiformis endodextranase (BAA13598); IMTD, B. fuscum var. dextranlyticum isomaltotriodextranase (BAA76382); SMCU-DEX, P. pinophilum SMCU3-14 dextranase; pUDEX, P. minioluteum HI-4 endodextranase (AAB47720); IPUA, A. niger isopullulanase (BAA18971).

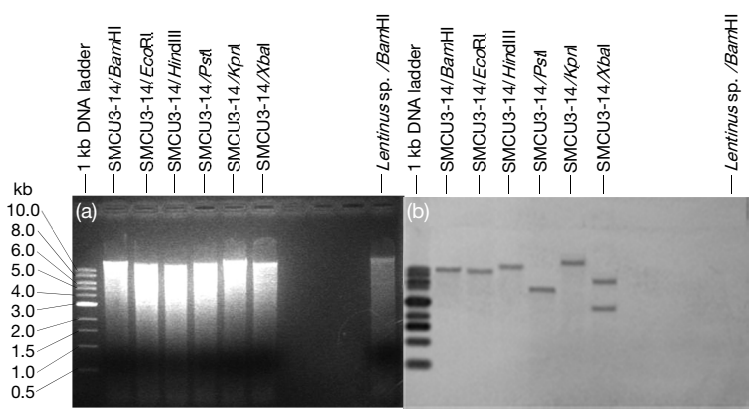

Fig. 4 (a) Agarose gel electrophoresis of the genomic DNA of $P$. pinophilum SMCU3-14 following complete restriction digestion with the indicated enzymes. The first lane is a 1-kb DNA ladder, and the last lane is the genomic DNA from Lentinus sp. digested with Bam $\mathrm{HI}$ as a negative control. (b) Southern hybridization with the digoxigeninlabelled full-length $S M C U$-DEX probe. The positions of the molecular markers (in bp) are indicated on the left.

with the empty pETHIS vector as a control were harvested and subjected to SDS-PAGE and blue dextranSDS-PAGE. SDS-PAGE stained with Coomassie brilliant blue R250 revealed an extra band with an estimated $\mathrm{M}_{\mathrm{W}}$ of approximately $66 \mathrm{kDa}$ in the IPTGinduced culture of the pNAT2 transformant. A corresponding dextranase activity band was also observed in the SDS-PAGE containing blue dextran zymograph (Fig. 6). Thus E. coli cells harbouring pNAT2 produced an active dextranase with a molecular mass of approximately $66 \mathrm{kDa}$.

\section{DISCUSSION}

Dextranases are found in a wide variety of fungi, bacteria, yeast, and Actinomyces, with fungi being the most important commercial sources ${ }^{15,29}$. However, only eight dextranase genes from five species of filamentous fungi plus one species of yeast have been reported to date, namely the dextranase genes from P. funiculosum (AJ272066), Penicillium marneffei ATCC 18224 (XM_002152550), P. minioluteum

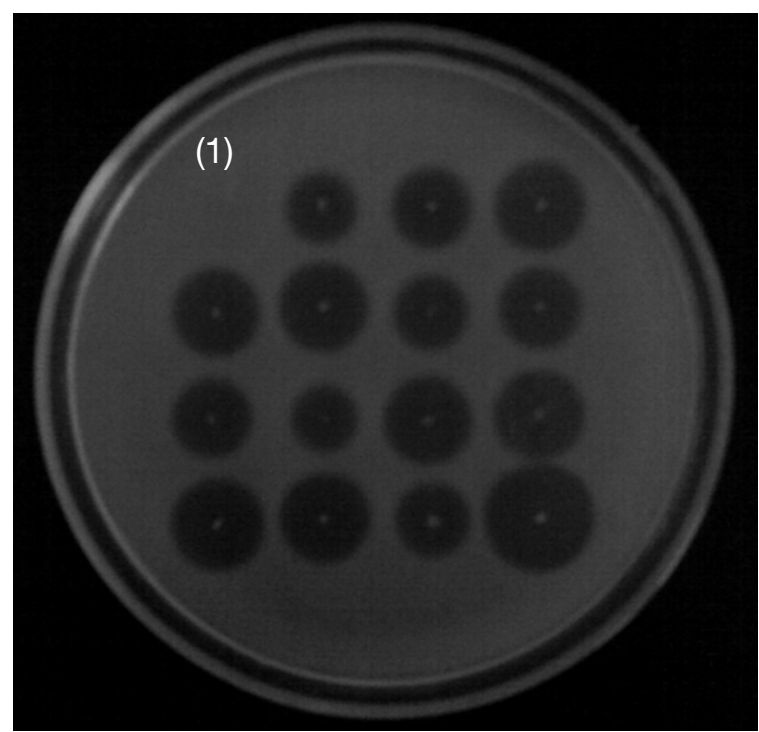

Fig. 5 LB agar plates with $1 \%(w / v)$ dextran after $18 \mathrm{~h}$ of culture with pNAT2- or the empty pETHIS vectortransformed E. coli colonies and then overlaid with $95 \%$ (v/v) ethanol. All the pNAT2-transformed E. coli colonies showed a large clear zone of dextran hydrolysis, whereas colonies of the empty vector transformant (indicated by (1)) did not.

(L41562, DQ394070 and AF020619), Verticillium alboatrum VaMs.102 (XM_003005140), V. dahliae VdLs.17 (DS572701), and the yeast L. starkeyi (AY520537). These fungal dextranases all belong to the GH49 family ${ }^{19}$. In this study, the cloning and sequencing of a GH49 dextranase from $P$. pinophilum SMCU3-14 and its heterologous expression in an alternative bacterial host were reported. The dextranase from P. pinophilum SMCU3-14 was found to be quite similar in amino acid sequence to all other currently known fungal dextranases. This is the first report of a dextranase gene sequence from P. pinophilum.

Sequence analysis of the complete dextranase gene (SMCU-DEX) revealed a single ORF of $1824 \mathrm{bp}$ without any intron. Four putative initiation codons 


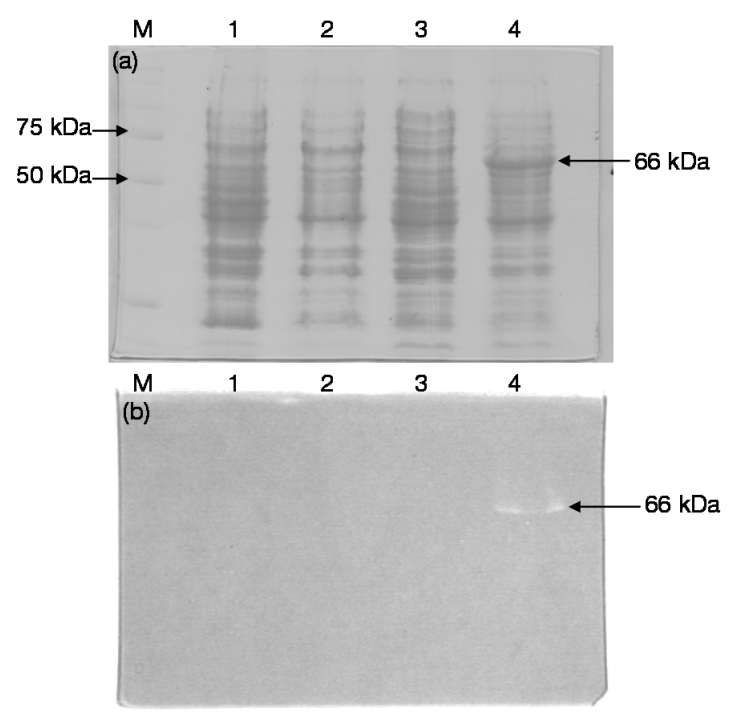

Fig. 6 SDS-PAGE analysis of the total protein and SDSPAGE-blue dextran zymograph analysis of the dextranase activity in the total protein sample. (a) Protein samples were separated by SDS-PAGE (10\% (w/v) acrylamide resolving gel) and then stained with Coomassie brilliant blue R250. (b) Protein samples were separated by SDS-PAGE containing $0.5 \%(\mathrm{w} / \mathrm{v})$ blue dextran and incubated for $\sim 18 \mathrm{~h}$ at $55^{\circ} \mathrm{C}$ for renaturation and dextranase activity. Dextranase activity is observed as a white band on a blue background. M: standard of the indicated molecular masses; lanes 1-4: protein samples from Rosetta-gami B (DE3)pLysS transformed with (lanes 1,2) pETHIS or (lanes 3, 4) pNAT2 and (lanes 1,3) non-induced or (lanes 2,4) induced with IPTG.

were found in the ORF, all in the same frame and coding for the same protein that (before maturation) would differ only in the length of the N-terminus (by up to 34 amino acids) and the presence $\left(\mathrm{ATG}_{1 / 2}\right)$ or absence $\left(\mathrm{ATG}_{3 / 4}\right)$ of an $\mathrm{N}$-terminal signal sequence. Although most fungal genes have several potential initiation codons, translation typically begins at the first $\mathrm{ATG}^{28}$. The ORF of $S M C U$-DEX starting from the first ATG $\left(\mathrm{ATG}_{1}\right)$ encodes a predicted protein of 608 amino acids (SMCU-DEX) with a predicted $\mathrm{M}_{\mathrm{W}}$ of approximately $66 \mathrm{kDa}$. However, it is also possible that the translation of the dextranase gene might begin from the second ATG $\left(\mathrm{ATG}_{2}\right)$ because the sequence preceding that codon $\left(\mathrm{CACA} A T G_{2}\right)$ resembles the consensus sequence for translation initiation identified in 99 genes of filamentous fungi $\left(5^{\prime} \mathrm{CAC} / \mathrm{AA} / \mathrm{CATG}_{2}\right.$ $\left.3^{\prime}\right)^{30}$. If the translation of the dextranase gene begins at the second ATG $\left(\mathrm{ATG}_{2}\right)$ codon, this gene would encode a protein of 605 amino acids with an $\mathrm{M}_{\mathrm{W}}$ of approximately $65 \mathrm{kDa}$. Regardless, both trans- lational products would be predicted to have an $\mathrm{N}$ terminal signal sequence and would yield the same mature protein of 588 amino acids and a molecular weight of $\sim 64 \mathrm{kDa}$ upon cleavage. The predicted presence of the signal peptide was expected given that dextranase is a secreted protein. Thus although not conclusive, our results suggest that the dextranase protein from $P$. pinophilum SMCU3-14 is synthesized from the first (or perhaps second) ATG codon as a preproenzyme, as the other two codons would not include the secretory signal peptide in the polypeptide sequence. The predicted mature polypeptide $\left(\mathrm{M}_{\mathrm{W}}\right.$ of $\sim 64 \mathrm{kDa}$ ) is smaller than that observed in the present study by SDS-PAGE $\left(\mathrm{M}_{\mathrm{W}} \sim 66 \mathrm{kDa}\right)$, suggesting posttranslational modification in the bacterial host or a failure to cleave the signal peptide.

We found that E. coli Rosetta-gami B (DE3)pLysS cells harbouring pNAT2 likely secreted active dextranase into the medium. Although heterologous protein expression in E. coli is mainly transported into the periplasmic space ${ }^{31}$, the potential secretion of recombinant dextranase observed in this study may occur due to the native secretion signal of SMCU-DEX present in the construct being operatively functional in E. coli. Regardless, the activity of dextranase from E. coli was confirmed in both agar diffusion assays and blue dextran-SDSPAGE zymographs. Future studies will seek to optimize the production and secretion of dextranase. Additionally, the subsequent optimization of largescale expression and purification would be required for the enzyme to be of commercial interest.

Since the application of native-produced fungal dextranases in the food and dental health industries might create some difficulty for obtaining approval from consumer or regulatory agents, the heterologous production of recombinant dextranase, such as in bacteria, as in this work, or in acceptable yeasts, may be cost-effective in medical and industrial applications. Another research is ongoing to express the enzyme in the yeasts Saccharomyces cerevisiae and Pichia pastoris, which are publicly acceptable for use in the food industry and may offer higher production yields and/or specific activities.

Acknowledgements: This study was financially supported by a $\mathrm{CU}$ graduate school thesis grant.

\section{REFERENCES}

1. Lee JM, Fox PF (1985) Purification and characterization of Paecilomyces lilacinus dextranase. Enzym Microb Tech 7, 573-7.

2. Bashari M, Tounkara F, Abdelhai MH, Lagnika C, Xu XM, Jin ZY (2013) Impact of dextranase on sugar 
manufacturing and its kinetic on the molecular weights of remaining dextran. Sugar Tech 15, 84-93.

3. Cuddihy Jr JA, Porro ME, Rauh JS (2001) The presence of total polysaccharides in sugar production and methods for reducing their negative effects. $J$ Am Soc Sugar Cane Tech 21, 73-91.

4. Gibbons RJ, van Houte J (1975) Dental caries. Аnnu Rev Med 26, 121-36.

5. Paster BJ, Boches SK, Galvin JL, Ericson RE, Lau CN, Levanos VA, Sahasrabudhe A, Dewhirst FE (2001) Bacterial diversity in human subgingival plaque. $J$ Bacteriol 183, 3770-83.

6. Pleszczyńska M, Szczodrak J, Rogalski J, Fiedurek J (1997) Hydrolysis of dextran by Penicillium notatum dextranase and identification of final digestion products. Mycol Res 101, 69-72.

7. Koenig D, Day D (1989) The purification and characterization of a dextranase from Lipomyces starkeyi. Eur J Biochem 183, 161-7.

8. Eggleston G, Dilks A, Blowers M, Winters K (2011) Successful application of dextranase in sugar beet factories. In: Proceedings of the American Society of Sugarbeet Technologists Meeting, New Mexico, 1-16.

9. Suganuma N, Anzai M, Takada N, Ichikawa H (1986) Dentifrice composition, Lion Corporation, USA Patent No. 4576816.

10. Tsuchiya R (2002) Plaque-inhibiting oral compositions, Novozymes, USA Patent No. 6413501.

11. Wu DT, Zhang HB, Huang LJ, Hu XQ (2011) Purification and characterization of extracellular dextranase from a novel producer, Hypocrea lixii F1002, and its use in oligodextran production. Process Biochem 46, 1942-50.

12. Mghir AS, Cremieux AC, Jambou R, Muffat-Joly M, Pocidalo JJ, Carbon C (1994) Dextranase enhances antibiotic efficacy in experimental viridans streptococcal endocarditis. Antimicrob Agents Chemother 38, 953-8.

13. Hattori A, Ishibashi K (1981) Screening of dextranase producing microorganisms. Agr Biol Chem 45, 2347-9.

14. Esawy MA, Mansour SH, Ahmed EF, Hassanein NM, El Enshasy HA (2012) Characterization of extracellular dextranase from a novel halophilic Bacillus subtilis NRC-B233b a mutagenic honey isolate under solid state fermentation. Eur J Chem 9, 1494-510.

15. Beldarraín A, Acosta N, Betancourt L, González LJ, Pons T (2003) Enzymic, spectroscopic and calorimetric studies of a recombinant dextranase expressed in Pichia pastoris. Biotechnol Appl Biochem 38, 211-21.

16. Aoki H, Sakano Y (1997) A classification of dextranhydrolysing enzymes based on amino-acid-sequence similarities. Biochem J 323, 859-61.

17. Suzuki N, Kim YM, Fujimoto Z, Momma M, Okuyama M, Mori H, Funane K, Kimura A (2012) Structural elucidation of dextran degradation mechanism by Streptococcus mutans dextranase belonging to glycoside hydrolase family 66. J Biol Chem 287, 19916-26.

18. Larsson AM, Andersson R, Stahlberg J, Kenne L, Jones
TA (2003) Dextranase from Penicillium minioluteum: reaction course, crystal structure, and product complex. Structure 11, 1111-21.

19. Li X, Millson SH, Coker RD, Evans IH (2006) Cloning and expression of Penicillium minioluteum dextranase in Saccharomyces cerevisiae and its exploitation as a reporter in the detection of mycotoxins. Biotechnol Lett 28, 1955-64.

20. Kang H-K, Kim SH, Park J-Y, Jin X-J, Oh D-K, Soo Kang S, Kim D (2005) Cloning and characterization of a dextranase gene from Lipomyces starkeyi and its expression in Saccharomyces cerevisiae. Yeast 22, 1239-48.

21. Noppornpanth S (1995) Strain improvement for the dextranase production of Penicillium sp. 61. MSc thesis, Chulalongkorn Univ.

22. Sangwichian E (1988) Dextranase from Penicillium sp. 61. MSc thesis, Chulalongkorn Univ.

23. Sambrook J, Russell DW (2001) Molecular Cloning: A Laboratory Manual, 3rd edn, Cold Spring Harbor Laboratory Press, New York.

24. Reader U, Broda P (1985) Rapid preparation of DNA from filamentous fungi. Lett Appl Microbiol 1, 17-20.

25. Fukumoto J, Tsuji H, Tsuru D (1971) Studies on mold dextranases. I. Penicillium luteum dextranase: its production and some enzymatic properties. J Biochem 69, 1113-21.

26. Laemmli UK (1970) Cleavage of structural proteins during the assembly of the head of bacteriophage T4. Nature 227, 680-5.

27. Barrett JF, Curtiss R (1986) Renaturation of dextranase activity from culture supernatant fluids of Streptococcus sobrinus after sodium dodecylsulfate polyacrylamide gel electrophoresis. Anal Biochem 158, 365-70.

28. Garcia B, Margolles E, Roca H, Mateu D, Raices M, Gonzales ME, Herrera L, Delgado J (1996) Cloning and sequencing of a dextranase-encoding cDNA from Penicillium minioluteum. FEMS Microbiol Lett 143, 175-83.

29. Sidebotham RL (1974) Dextrans. Adv Carbohydr Chem Biochem 30, 371-444.

30. Ballance DJ (1991) Transformation systems for filamentous fungi and an overview of fungal gene structure. In: Leong SA, Berka RM (eds) Molecular Industrial Mycology. Systems and Applications for Filamentous Fungi, Marcel Dekker, New York, pp 1-29.

31. Khalikova E, Susi P, Korpela T (2005) Microbial dextran-hydrolyzing enzymes: fundamentals and applications. Microbiol Mol Biol Rev 69, 306-25. 